least 20\%. The SEs of the MSM associations were in median $19.4 \%$ greater than the respective conventional SEs (IOR: $2.4 \%$ to $47.5 \%$ ) in the 156 available associations. In 88 papers, MSMs were used to analyse real data; only $53(60.2 \%)$ of these papers reported that stabilised inverse-probability weights (IPWs) were used, and only 28 $(31.8 \%)$ reported that they verified that the mean of the stabilised IPWs was close to one.

Conclusions We found important differences between MSMs and conventional models in real studies. Reporting of MSMs can be improved.

\section{P1-55 COMPARISON OF TWO CRITERIA TO EVALUATE PHYSICAL ACTIVITY PATTERN IN ADULTS}

doi:10.1136/jech.2011.142976c.48

${ }^{1,2} \mathrm{C}$ S Suzuki, ${ }^{2} \mathrm{~S}$ A de Moraes, ${ }^{2} \mathrm{C}$ C M de Freitas. ${ }^{1}$ Universidade Estadual do Centro Oeste, Irati, Parana, Brazil, ${ }^{2}$ University of Sao Paulo, Ribeirao Preto, Sao Paulo, Brazil

Background The international literature has presented several criteria for classifying people in terms of physical activity pattern, making it difficult to compare the results.

Objective The study aims to evaluate the reliability between two criteria used to classify physical activity pattern, in adults living in Ribeirão Preto, SP, Brazil, in 2006.

Methods Using data from a cross-sectional population-based study, the International Physical Activity Questionnaire (IPAQ) and the American College of Sports Medicine/American Heart Association (ACSM/AHA) criteria were applied. The $\kappa$ statistic estimated by point and $95 \%$ CIs, stratified by gender, age, education level and income was applied to evaluate reliability between the two criteria. Results The results showed an agreement close to the unity $(\kappa \approx 1)$ between the two criteria, in both genders, with crude coefficients $\mathrm{k}_{\text {male }}=0.95$ (95\% CI 0.83 to 1.06$)$ and $\mathrm{k}_{\text {female }}=0.93$ (95\% CI 0.85 to 1.01). The lowest magnitude of $\kappa$ were found in the age group of 60 years and over in both genders $\left[k_{\text {male }}=0.87\right.$ (95\% CI 0.60 to 1.15); $\mathrm{k}_{\text {female }}=0.88$ (95\% CI 0.70 to 1.06)], and among illiterate women or with $<01$ year of education $[k=0.72$ (95\% CI 0.34 to 1.09)].

Conclusions The IPAQ and ACSM/AHA criteria showed practically the same ability to classify participants in terms of physical activity levels, allowing comparison of population-based studies that have used the IPAO instrument to obtain information about physical activity practice. The results pointed out the higher prevalence of "actives" when the second criteria was applied and "walking" was the main practice of physical activity.

\section{P1-56 UNDERSTANDING THE NATURAL PROGRESSION IN FEV DECLINE IN PATIENTS WITH LUNG DISEASE}

doi:10.1136/jech.2011.142976c.49

${ }^{1} \mathrm{D}$ Taylor-Robinson, ${ }^{*}{ }^{2} \mathrm{M}$ Whitehead, ${ }^{1} \mathrm{H}$ V Olesen, ${ }^{3} \mathrm{~T}$ Pressler, ${ }^{4} \mathrm{~F}$ Diderichsen, ${ }^{1} \mathrm{R}$ Smyth, ${ }^{5} \mathrm{P}$ Diggle. ${ }^{1}$ University of Liverpool, Liverpool, UK; ${ }^{2}$ Aarhus University Hospital, Aarhus, Denmark; ${ }^{3}$ Cystic Fibrosis Centre, Rigshospitalet, Copenhagen, Denmark; ${ }^{4}$ University of Copenhagen, Copenhagen, Denmark; ${ }^{5}$ Chicas, Lancaster University, Lancaster, UK

Introduction We outline a novel approach for longitudinal modelling of lung function with long-term follow-up in which within-patient variation over time is described by a stationary (mean-reverting) stochastic process, and apply these techniques to a unique dataset of cystic fibrosis patients in Denmark. The aim is to quantify how lung function changes in chronic lung diseases.

Methods The Danish CF register contains data collected on a monthly basis with up to 30 years of follow-up. Our statistical analysis framework is that of a linear mixed effects model with longitudinally structured correlation. Using open-source software we describe how to partition the variability in the data into three components (between and within patient, and measurement error) using the empirical variogram. A parametric model for lung function decline can then be developed. We apply this approach to explore the effect of age, birth cohort and infection status on lung function decline.

Results The dataset contains 70448 measures on 479 patients seen between 1960 and 2009. The empirical variogram shows slowly decaying long-term correlation ( $>15$ years) in FEV1, with half of the variability in lung function explained by within person variation. The mean rate of lung function decline is $0.96 \%$ per year $(95 \% \mathrm{CI}$ 0.86 to 1.07). There is a significant cohort effect, and chronic infection significantly increases the rate of lung function decline.

Conclusions We apply a novel modelling approach to demonstrate that lung function in early life in the Danish cystic fibrosis population is correlated with lung function over 15 years later.

\section{P1-57 USING PARTIAL LEAST SQUARES REGRESSION FOR THE AGE-PERIOD-COHORT ANALYSIS}

doi:10.1136/jech.2011.142976c.50

${ }^{1} \mathrm{Y} \mathrm{K} \mathrm{Tu},{ }^{*} \mathrm{~K}$ L Chien, ${ }^{1} \mathrm{M}$ Gilthorpe. ${ }^{1}$ University of Leeds, Leeds, UK; ${ }^{2}$ National Taiwan University, Taipei, Taiwan

Background Identification has been a problem with the Age-PeriodCohort analysis. Since Age + Cohort $=$ Period, there is no unique solution using generalised linear modelling. To overcome this problem of perfect collinearity we propose to use partial least squares regression (PLSR), a dimension-reduction technique widely used in bioinformatics. Data from a large Taiwanese cohort was used to illustrate our approach.

Methods PLSR is a set of algorithms that aims to maximise the covariance between outcome and successively extracted orthogonal components under the constraint that the sum of squared weights is equal to unity. To assess the impact of age, birth year and year of examination on the levels of metabolic syndrome (MetS) components, we used PLSR to analyse data collected by Mei-Jaw clinics in Taiwan in years 1996 and 2006. Confounders, such as the number of years in formal education, alcohol intake, smoking history status, and betel-nut chewing were adjusted for.

Results As the age of individuals increased, the values of components generally increased. People born after 1970 had lower fasting plasma glucose, lower body mass index, lower diastolic blood pressure, lower triglycerides, lower low-density cholesterol lipids and greater high-density cholesterol lipids. A similar pattern between the trend in levels of metabolic syndrome components against birth year of birth and economic growth in Taiwan were also found.

Conclusions Our study found cohort effects in some MetS components, suggesting associations between the changing environment and health outcomes in later life. PLSR provides a flexible analytical strategy for the Age-Period-Cohort analysis.

\section{P1-58 THE VOLUMETRIC MEASUREMENT OF BRAIN IMAGING BIOMARKERS FOR EPIDEMIOLOGICAL STUDIES CAN BE MISLEADING IN THE VALIDATION OF IMAGE SEGMENTATION METHODS}

doi:10.1136/jech.2011.142976c.51

M C Valdes-Hernandez,* N A Royle, S Munoz-Maniega, M E Bastin, I J Deary, J M Wardlaw. University of Edinburgh, Edinburgh, UK

Large scale neuro-epidemiology studies using brain imaging need tissue segmentation methods to determine lesion or normal tissue 